\title{
Long-lived staus from cosmic rays
}

\author{
Markus Ahlers ${ }^{1}$, José Ignacio Illana ${ }^{2}$, Manuel Masip ${ }^{2}$ and \\ Davide Meloni ${ }^{3}$ \\ ${ }^{1}$ Deutsches Elektronen-Synchrotron DESY, Notkestraße 85, D-22607 Hamburg, \\ Germany \\ ${ }^{2}$ CAFPE and Departamento de Física Teórica y del Cosmos, Universidad de \\ Granada, 18071 Granada, Spain \\ ${ }^{3}$ INFN and Dipartimento di Fisica, Università degli Studi di Roma \\ 'La Sapienza', 00185 Rome, Italy \\ E-mail: markus.ahlers@desy.de, jillana@ugr.es, masip@ugr.es, \\ meloni@roma1.infn.it and Davide.Meloni@roma1.infn.it
}

Received 4 June 2007

Accepted 19 July 2007

Published 7 August 2007

Online at stacks.iop.org $/ \mathrm{JCAP} / 2007 / \mathrm{i}=08 / \mathrm{a}=008$

doi:10.1088/1475-7516/2007/08/008

\begin{abstract}
The collision of a high energy cosmic ray with a nucleon in the upper atmosphere could produce long-lived heavy particles. Such particles would be very penetrating, since the energy loss in matter scales as the inverse mass, and could reach a neutrino telescope like IceCube from large zenith angles. Here we study this possibility and focus on the long-lived stau of supersymmetric models with a gravitino lightest supersymmetric particle. The signal would be a pair of muon-like parallel tracks separated by $50 \mathrm{~m}$ along the detector. We evaluate the background of muon pairs and show that any events from zenith angles above $80^{\circ}$ could be explained by the production of these heavy particles by cosmic rays. For light squark and gluino masses the number of these events in cubic kilometer neutrino telescopes is of the order of one per year.
\end{abstract}

Keywords: cosmic rays detectors, cosmic rays

ArXiv ePrint: 0705.3782 


\section{Contents}

1. Introduction 2

2. Production of stau pairs 3

3. Background of muon pairs and signal at IceCube 6

4. Summary and discussion $\quad 8$

$\begin{array}{ll}\text { Acknowledgments } & 9\end{array}$

References $\quad 9$

\section{Introduction}

The hierarchy problem has motivated an intensive search for new physics during the past 20 years. Colliders like LEP, the Tevatron, or the B factories have explored the standard model (SM) at the quantum level but have not reached the energy or the sensitivity necessary to detect new physics. There is experimental evidence for neutrino masses, whereas cosmological data strongly suggest the presence of a stable weakly interacting massive particle (WIMP) as the origin of the dark matter of the universe. However, these features could be easily added to the SM without changing its structure. Therefore, as we approach the search for extensions like supersymmetry (SUSY), technicolor or extra dimensions at the LHC, it is clear that we should never underestimate the SM.

On the other hand, cosmic rays are another source of elementary particles of very high energy with the potential to explore the physics beyond the SM. When a proton of $10^{8} \mathrm{GeV}$ from outer space hits an atmospheric nucleon it provides a center of mass energy $\sqrt{s}=\sqrt{2 m_{N} E}$ around $14 \mathrm{TeV}$. A small fraction of these protons (or of the secondary particles with still enough energy) may then produce exotic massive particles. Of course, the question is whether such an event could give any observable signal. In this paper we argue that this is the case, the long-lived charged particles present in some extensions of the SM could provide a distinct signature when they cross a neutrino telescope from large zenith angles.

The process that we propose could take place in SUSY models with an exact $R$-parity, a gravitino lightest SUSY particle (LSP) working as dark matter, and a charged next-toLSP (NLSP) [1]-[11]. The collision of the cosmic proton with the nucleon could produce any pair of SUSY particles, which would then decay promptly into the NLSP. Since the NLSP couples very weakly to the LSP gravitino, it will be long-lived and able to cross a kilometer-long detector like IceCube. The generic features of this framework could be also found in other extensions of the SM, like Little Higgs models [12]. These models may incorporate a $T$-parity [13] separating the standard and the exotic particles. The $T$-parity would forbid unobserved mixing between both sectors, tree-level four-fermion operators, and would also make the lightest particle in the odd sector stable. If this particle (constituting the dark matter) is very weakly coupled with the rest, the next-tolightest one will be long-lived. 
Table 1. Constants defining the total cross section with the air $\sigma_{T}^{\text {ha }} \approx C_{0}^{h}+$ \begin{tabular}{lcll}
\multicolumn{2}{l}{$C_{1}^{h} \ln (E / \mathrm{GeV})+C_{2}^{h} \ln ^{2}(E / \mathrm{GeV})}$. \\
\hline$h$ & $C_{0}^{h}(\mathrm{mb})$ & $C_{1}^{h}(\mathrm{mb})$ & $C_{2}^{h}(\mathrm{mb})$ \\
\hline$N$ & 185.7 & 13.3 & 0.08 \\
$\pi$ & 100.5 & 16.9 & 0.00 \\
$K$ & 79.7 & 13.9 & 0.05 \\
\hline
\end{tabular}

The possibility to observe quasi-stable gluinos in IceCube [14] has been considered in $[15,16]$ in the framework of split-SUSY models with very heavy sfermions [17]. Here we will focus on non-colored particles, which present some remarkable differences with the gluinos. In particular, as one of these particles propagates in matter it loses energy at a much smaller rate than an $R$-hadron $[18,19]$. This fact makes it easier to confuse it with a muon, but it also lets the particle reach IceCube form larger zenith angles. To be definite we will consider a long-lived stau $\widetilde{\tau}_{R}{ }^{4}$, although our arguments would be analogous for any massive charged particle: charginos, other sleptons, or vector-like leptons that may appear in Little Higgs models.

Other analyses of the production of exotic particles by cosmic rays refer to primary neutrinos [21]-[24]. Being weakly interacting, the relative effect of new physics on the neutrino-nucleon cross section may be larger (see below), however, it is difficult to make precise predictions until the flux of cosmic neutrinos is determined. In contrast, our analysis here relies on a flux of primary cosmic rays that is well known in the relevant energy region $10^{5}-10^{8} \mathrm{GeV}$.

The outline of the paper is as follows. In section 2, we calculate the rate of long-lived stau pairs produced by collisions of primary and secondary cosmic rays in the atmosphere. We discuss then in section 3 the signal of these pairs at IceCube and the background from muon pairs. Section 4 includes a summary of our results.

\section{Production of stau pairs}

At energies above $10^{4} \mathrm{GeV}$ the decay length of nucleons, charged pions and kaons is much larger than their interaction length in the air. Therefore, as they propagate in the atmosphere the probability that one of these hadrons $(h)$ collides with a nucleon $(N)$ to produce new physics is just [25]

$$
\mathcal{P}_{X}^{h}(E) \approx \frac{A \sigma_{X}^{h N}}{\sigma_{\mathrm{T}}^{\mathrm{ha}}} .
$$

In this expression $\sigma_{X}^{h N}$ is the cross section to produce the exotic particle(s) $X$ and $\sigma_{\mathrm{T}}^{\text {ha }}$ the total cross section of the hadron with the air (we assume $A=14.6$ nucleons in a nucleus of air and neglect nuclear effects). The (default) cross sections with the air used by CORSIKA [26] above $10^{4} \mathrm{GeV}$ can be approximated by $\sigma_{\mathrm{T}}^{\text {ha }} \approx C_{0}^{h}+C_{1}^{h} \ln (E / \mathrm{GeV})+$ $C_{2}^{h} \ln ^{2}(E / \mathrm{GeV})$, with the constants given in table 1 . Since $\sigma_{\mathrm{T}}^{\text {ha }}$ is above $100 \mathrm{mb}$, it is

\footnotetext{
${ }^{4}$ We will assume in the following that the $\widetilde{\tau}_{R}$ lifetime is much larger than the propagation time through the Earth (see e.g. [20]).
} 


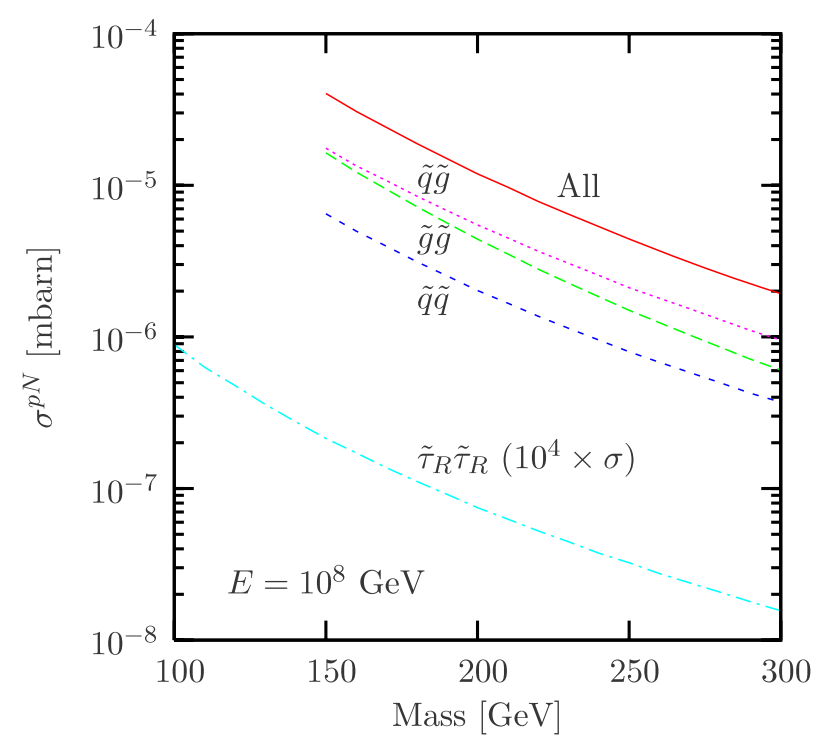

Figure 1. $p N$ cross section to produce SUSY particles for different values of the stau and the (common) squark/gluino mass and a proton energy of $10^{8} \mathrm{GeV}$.

apparent that this probability will be very small and that it would be much larger for a neutrino propagating in matter.

The cross section to produce SUSY particles in a hadronic collision depends basically on their mass and on whether they have strong interactions. All the cross sections at the parton level can be found in [27]. Collider bounds on SUSY particles with prompt decay into a neutral LSP are around $250 \mathrm{GeV}$ for gluinos and squarks, and $100 \mathrm{GeV}$ for the stop, the sbottom, charginos, and charged sleptons [28]. These bounds, however, may not apply if the particles decay instead into a long-lived charged or colored SUSY particle. For example, in order to minimize the SM background a recent analysis [29] of jets plus missing momentum at the run II of the Tevatron imposes a veto on events with an isolated electron or muon with large transverse momentum. However, gluino or squark events will include here final staus (instead of neutralinos) that could be taken by isolated muons. We are not aware of specific bounds on the colored SUSY spectrum in stau NLSP models. Notice that bounds based on the delay in the time of flight versus a muon or the anomalous ionization of the staus should also be specific, as they are based on the absence of slow-moving $(\beta \leq 0.6)$ charged particles, but here the staus get an extra boost from the decay of the parent squark or gluino. Through the paper we will then consider slepton, chargino and neutralino masses as low as $100 \mathrm{GeV}$ and colored SUSY particles above $150 \mathrm{GeV}$.

In figures 1 and 2 we plot the total $h N(h=p, \pi)$ cross sections to produce pairs of these SUSY particles for different SUSY masses (left panel) and for values of the hadron energy between $10^{4}$ and $10^{11} \mathrm{GeV}$ (right panel). We have used the CTEQ6M [30] (MRSS [31]) parton distribution functions for baryonic (mesonic) interactions, with the renormalization scale $\mu=0.2 m_{\tilde{X}}$ suggested by a NLO calculation [32]. We observe that the cross section to produce directly a pair of long-lived staus of $100 \mathrm{GeV}$ is much smaller than via the production and prompt decay of colored particles of mass around $200 \mathrm{GeV}$. 


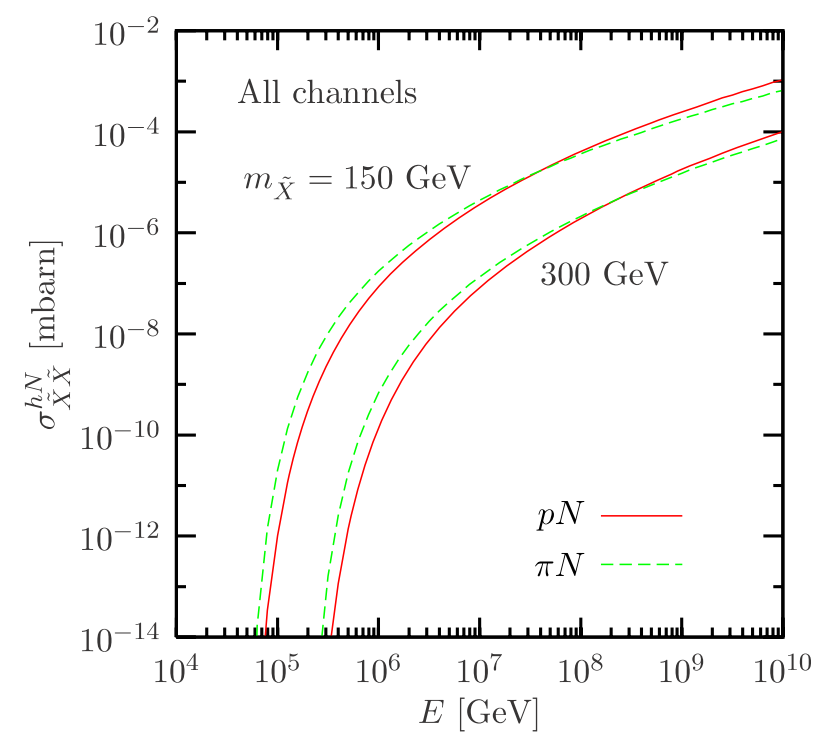

Figure 2. Cross section to produce any pair of colored SUSY particles in a $p N$ and a $\pi N$ collision for different incident energies and a squark/gluino mass $m_{\widetilde{X}}=150,300 \mathrm{GeV}$.

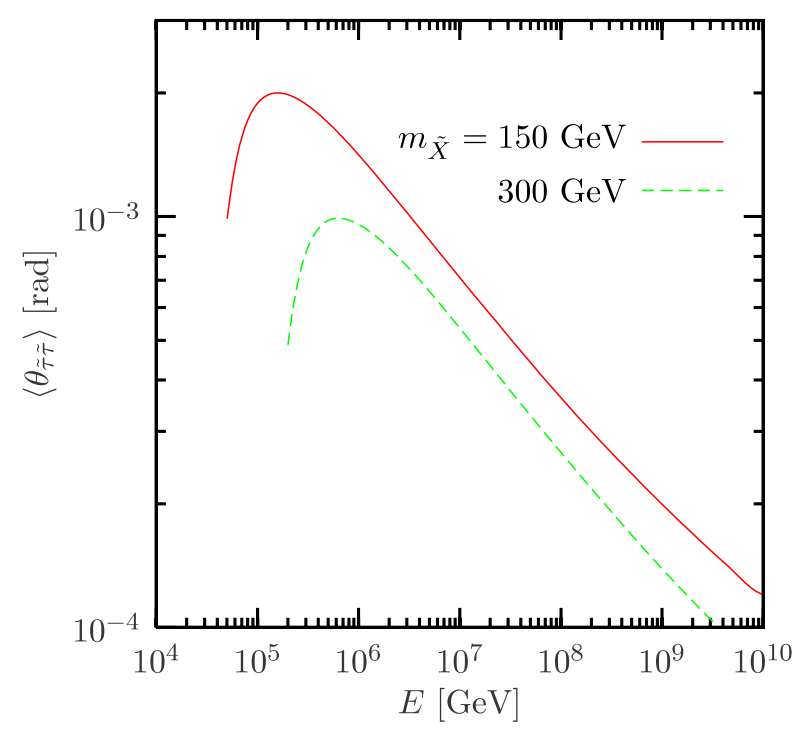

Figure 3. Average angle between the two staus (in the laboratory frame) for $m_{\widetilde{X}}=150,300 \mathrm{GeV}$.

In the latter case, we estimate that the final stau will carry a fraction $\eta$

$$
\eta \approx \frac{m_{\widetilde{X}}^{2}+m_{\widetilde{\tau}}^{2}}{2 m_{\widetilde{X}}^{2}} \quad(\tilde{X}=\widetilde{g}, \widetilde{q})
$$

of the energy of the parent gluino or squark. The average angle of the stau pair (in the lab frame) for different energies of an incident proton are given in figure 3. 


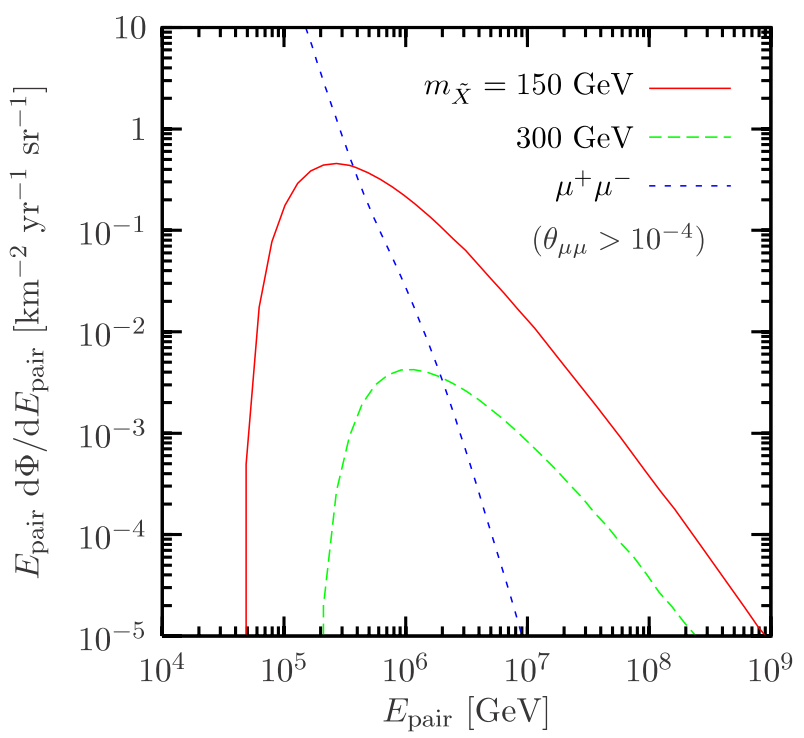

Figure 4. Flux of stau pairs produced by cosmic rays in terms of the (total) stau energy $E_{\tilde{\tau} \widetilde{\tau}}$ on production for $m_{\tilde{X}}=150,300 \mathrm{GeV}$ and $\eta=1$. We include the flux of muon pairs produced with an opening angle above $10^{-4} \mathrm{rad}$.

To evaluate the production rate of stau pairs by cosmic rays we need the total flux of hadrons: primary plus secondary nucleons, pions and kaons produced at any depth in the atmosphere and with enough energy to create staus in the collision with an air nucleon. This analysis has been carried out in [15] assuming a flux of primary nucleons

$$
\frac{\mathrm{d} \Phi_{N}}{\mathrm{~d} E} \approx 1.8\left(\frac{E}{1 \mathrm{GeV}}\right)^{-2.7} \frac{\text { nucleons }}{\mathrm{cm}^{2} \mathrm{~s} \mathrm{sr} \mathrm{GeV}},
$$

for energies up to $10^{6} \mathrm{GeV}$, with a spectral index that changes to 3 in the interval $10^{6}$ $10^{9} \mathrm{GeV}$ and goes back to 2.7 at higher energies (e.g. [33]). We will use in the following the results on the flux of secondary hadrons (nucleons, pions, and kaons) derived there.

The flux of quasi-stable staus produced via the prompt decay of a pair $\widetilde{X} \widetilde{X}^{\prime}$ of SUSY particles is then

$$
\Phi_{\tilde{\tau} \widetilde{\tau}}=\sum_{h=N, \pi, K} \int_{E_{\min }}^{\infty} \mathrm{d} E \frac{\mathrm{d} \Phi_{h}}{\mathrm{~d} E} \mathcal{P}_{\widetilde{X} \tilde{X}^{\prime}}^{h}(E) .
$$

In figure 4 we plot the differential flux $\left(\mathrm{d} \Phi / \mathrm{d} E_{\tilde{\tau} \tau}\right)$ of stau pairs produced by cosmic rays for squarks and gluinos masses of 150 and $300 \mathrm{GeV}$ and $\eta=1$.

\section{Background of muon pairs and signal at IceCube}

The flux of stau pairs produced high in the atmosphere needs to propagate down to the core of IceCube, about two kilometers under the antarctic ice, to be observed. In addition, the possible signal faces a strong background of muon pairs crossing the detector. We plot in figure 4 the flux $\left(\mathrm{d} \Phi / \mathrm{d} E_{\mu \mu}\right)$ of muon pairs ${ }^{5}$ produced by cosmic rays of energy

\footnotetext{
${ }^{5}$ We neglect the muons from tau decays as they are a $\approx 1 \%$ correction to this flux.
} 


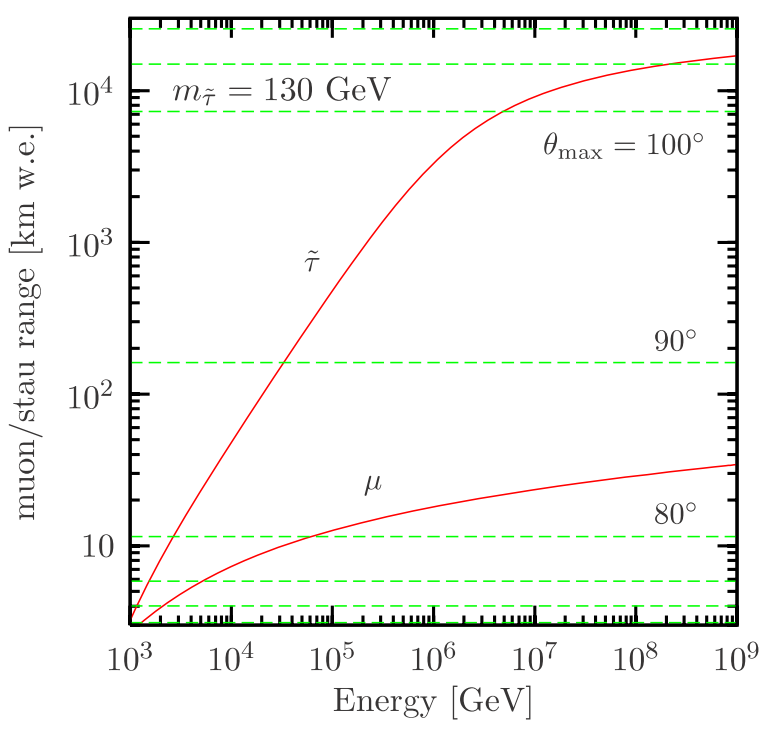

Figure 5. Range of staus and muons of different energy. The dashed lines show the integrated column depth of the Earth from the center of IceCube for increasing zenith angles $\left(\Delta \theta_{\max }=10^{\circ}\right)$.

$E_{\mathrm{h}}>10^{4} \mathrm{GeV}$. We include only the events where the two muons are produced with an opening angle above $10^{-4} \mathrm{rad}$, since smaller angles imply a separation between the two muon tracks that cannot be resolved at IceCube (see below). Notice that this requirement cuts off muon pairs with an invariant mass near threshold, $\sqrt{\hat{s}} \leq 1 \mathrm{GeV}$, where the PDFs are mostly unknown and the process would be better described in terms of hadronic resonances.

The propagation of muons and heavy charged particles in matter is well understood. For a muon of energy $E_{\mu}>2 m_{\mu}$ the mean energy loss per column density (measured in $\mathrm{g} \mathrm{cm}^{-2}$ ) can be approximated as

$$
-\frac{\mathrm{d} E_{\mu}}{\mathrm{d} z}=\alpha_{\mu}+\beta_{\mu} E_{\mu}
$$

where $\alpha_{\mu} \approx 2 \times 10^{-3} \mathrm{GeV} \mathrm{cm} \mathrm{g}^{-1}$ describes ionization effects and $\beta_{\mu} \approx 4 \times 10^{-6} \mathrm{~cm}^{2} \mathrm{~g}^{-1}$ accounts for bremsstrahlung, pair production and photohadronic processes. The solution of equation (5) provides an approximation of the total range of (initially) very relativistic muons, which we consider in the following 6 .

For a stau, at the lowest order ionization effects coincide $\left(\alpha_{\tilde{\tau}} \approx \alpha_{\mu}\right)$ whereas the other effects depend mainly on the boost factor of the particle, which implies $\beta_{\widetilde{\tau}} \approx \beta_{\mu} m_{\mu} / m_{\tilde{\tau}}$. For $m_{\tilde{\tau}} \approx 100 \mathrm{GeV}$, this means that a stau of energy above $10^{5} \mathrm{GeV}$ loses $10^{3}$ times less energy than a muon of the same energy, but below $500 \mathrm{GeV}$ they deposit energy at a similar rate. In our analysis we will use the approximation for the range of a stau provided in [18] and will neglect losses through electroweak interactions [19], as they are not important for the stau energies that we obtain.

In figure 5 we plot the range of staus and muons of energy between $10^{3}$ and $10^{9} \mathrm{GeV}$. We give the correspondence between integrated column depth of the Earth (see e.g. [34])

${ }^{6}$ Below $E \approx 2 m$ the ionization energy loss grows like $1 / \beta^{2}$ (see e.g. [28]). 


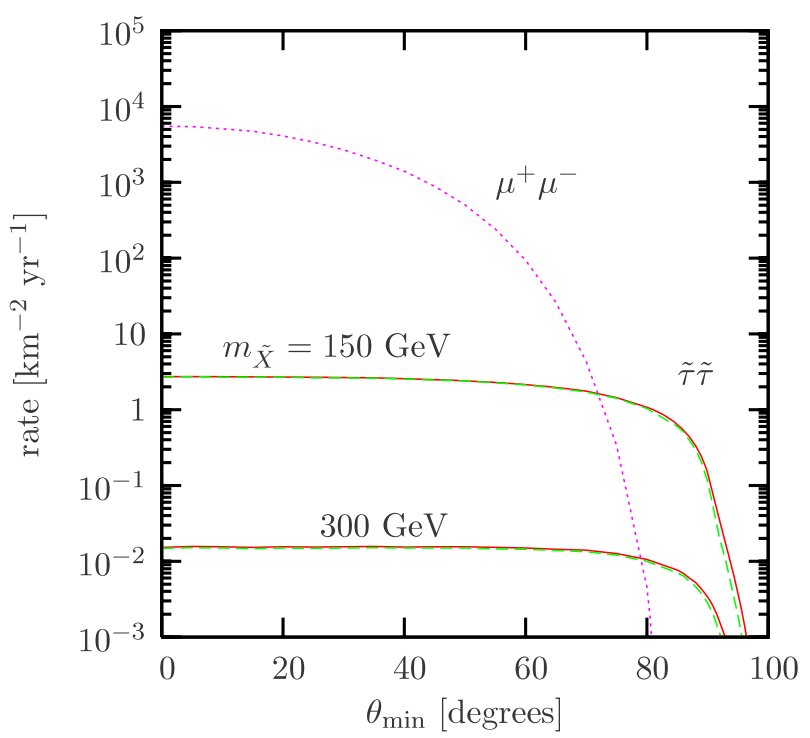

Figure 6. Integrated number of stau and muon pairs with a minimal separation of $50 \mathrm{~m}$ at the detector for different values of a minimal zenith angle.

and zenith angle for several trajectories ending at the center of IceCube. We observe, for example, that whereas a muon of $E=10^{7} \mathrm{GeV}$ has a range of about $25 \mathrm{~km}$ water equivalent (w.e.) and can reach IceCube from a zenith angle $\theta_{\max } \approx 86^{\circ}$, the range of a stau of the same energy is 360 times larger, which makes it able to reach IceCube from zenith angles of up to $\theta_{\max } \approx 105^{\circ}$.

Another relevant observable is the separation of the two particles when they cross IceCube. This depends on their angle at the creation point (see figure 3 ) and the distance from that point to the telescope. The interaction length of a $10^{7} \mathrm{GeV}$ proton in air is around $4 \mathrm{~g} \mathrm{~cm}^{-2}$ (its cross section is $\sigma_{\mathrm{T}}^{\text {ha }} \approx 400 \mathrm{mb}$ ), which corresponds to an altitude of about $20 \mathrm{~km}$ in the atmosphere. Therefore, if a primary proton creates a stau pair it will do it around that altitude. The production of a stau pair by a secondary hadron will typically occur along the second interaction length, finishing at around $15 \mathrm{~km}$, and so on. To estimate the distance between the parallel stau tracks at IceCube we will assume that they are created at a height $H \approx 15 \mathrm{~km}$. This implies that stau pairs coming from zenith angles of $60^{\circ}, 80^{\circ}$, and $100^{\circ}$ fly an approximate distance of about 30,90 , and $2300 \mathrm{~km}$, respectively, to reach the center of IceCube.

In figure 6 we compare the number of stau and muon pairs reaching IceCube with a separation larger than $50 \mathrm{~m}$, so that the two tracks can be resolved [35]. We plot the flux of these particles coming from zenith angles larger than the value given in the $x$ axis (e.g. $\theta_{\min }=0$ corresponds to pairs coming from any direction). We show the cases $m_{\tilde{X}}=150,300 \mathrm{GeV}$ and $\eta=1,0.7$. We observe that from zenith angles between $80^{\circ}$ and $95^{\circ}$ there is a possible signal with no background from muon pairs.

\section{Summary and discussion}

Cosmic rays may be continuously producing massive particles when they collide with nucleons in the upper atmosphere. If these particles are long-lived they will be able to 
reach a detector like IceCube, about $2 \mathrm{~km}$ under the ice. To have a sizable production rate (order 1 per year and square kilometer) the particles should be produced through strong interactions. This would be the case for a quasi-stable stau resulting from the prompt decay of a gluino or a squark. We have studied in some detail the possibility to observe such an event.

The heavy staus would be produced in pairs at altitudes around $15 \mathrm{~km}$, and as they approach IceCube the two staus would separate. In principle, they could be confused with a muon pair: a muon and a stau of $500 \mathrm{GeV}$ would give in IceCube a very similar signature. We have shown, however, that it is possible to reduce the dimuon background below the signal. Above $E \approx 500 \mathrm{GeV}$ muons lose energy in ice much faster than the staus. As a consequence, while muons will never reach IceCube from directions close to the horizon, staus can come from zenith angles of up to $110^{\circ}$. In addition, larger zenith angles mean also larger distance of flight and, in turn, larger separation between the two tracks at IceCube.

We obtain that any events with two muon-like tracks separated by more than $50 \mathrm{~m}$ coming from zenith angles of $80^{\circ}-100^{\circ}$ would be a clear signal of heavy charged particles produced by cosmic rays high in the atmosphere (see figure 6).

One may wonder if this signal could also be distinguished from possible stau pairs produced by primary neutrinos, which have been extensively considered in the literature. We find two main differences with such an event. Suppose that the primary comes horizontally $\left(\theta=90^{\circ}\right)$. In our case the staus will be created high in the atmosphere, whereas in a neutrino event the interaction to produce them will typically take place deep inside the ice. This implies a smaller distance between the two stau tracks along IceCube. A second important difference is that while neutrino events could come basically from any direction, the staus produced by primary protons vanish at zenith angles above about $115^{\circ}$.

We think that the study of inclined $\left(\theta \geq 60^{\circ}\right)$ two-muon events at IceCube would be interesting by itself. For example, it can be used as an indirect measure of the total (primary plus secondary) flux of hadrons of $10^{4}-10^{9} \mathrm{GeV}$ and, thus, as a test for the different codes that simulate extensive air showers. Going to zenith angles below the horizon the SM background vanishes, leaving some room for exotic physics.

\section{Acknowledgments}

We would like to thank Francisco del Águila, Fernando Cornet, Andreas Ringwald, and Christian Spiering for useful discussions. This work was supported by MEC of Spain (FPA2006-05294) and Junta de Andalucía (FQM-101 and FQM-437). We also acknowledge financial support from a grant CICYT-INFN (07-10).

\section{References}

[1] Ellis J R, Olive K A, Santoso Y and Spanos V C, 2004 Phys. Lett. B 5887 [SPIRES] [hep-ph/0312262]

[2] Buchmüller W, Hamaguchi K, Ratz M and Yanagida T, 2004 Phys. Lett. B 58890 [SPIRES] [hep-ph/0402179]

[3] Feng J L, Su S and Takayama F, 2004 Phys. Rev. D 70075019 [SPIRES] [hep-ph/0404231]

[4] Hamaguchi K, Kuno Y, Nakaya T and Nojiri M M, 2004 Phys. Rev. D 70115007 [SPIRES] [hep-ph/0409248]

[5] Feng J L and Smith B T, 2005 Phys. Rev. D 71015004 [SPIRES] [hep-ph/0409278]

[6] Hamaguchi K and Ibarra A, 2005 J. High Energy Phys. JHEP02(2005)028 [SPIRES] [hep-ph/0412229] 
[7] Brandenburg A, Covi L, Hamaguchi K, Roszkowski L and Steffen F D, 2005 Phys. Lett. B 61799 [SPIRES] [hep-ph/0501287]

[8] Steffen F D, 2006 J. Cosmol. Astropart. Phys. JCAP09(2006)001 [SPIRES] [hep-ph/0605306]

[9] Ellis J R, Raklev A R and Oye O K, 2006 J. High Energy Phys. JHEP10(2006)061 [SPIRES] [hep-ph/0607261]

[10] Cyburt R H, Ellis J, Fields B D, Olive K A and Spanos V C, 2006 J. Cosmol. Astropart. Phys. JCAP11(2006)014 [SPIRES] [astro-ph/0608562]

[11] Cakir O, Cakir I T, Ellis J R and Kirca Z, 2007 Preprint hep-ph/0703121

[12] Arkani-Hamed N, Cohen A G, Katz E and Nelson A E, 2002 J. High Energy Phys. JHEP07(2002)034 [SPIRES] [hep-ph/0206021]

[13] Cheng H-C and Low I, 2003 J. High Energy Phys. JHEP09(2003)051 [SPIRES] [hep-ph/0308199]

[14] Ahrens J et al (IceCube), 2004 Astropart. Phys. 20507 [SPIRES] [astro-ph/0305196] http://icecube.wisc.edu/

[15] Illana J I, Masip M and Meloni D, 2007 Phys. Rev. D 75055002 [SPIRES] [hep-ph/0611036]

[16] Hewett J L, Lillie B, Masip M and Rizzo T G, 2004 J. High Energy Phys. JHEP09(2004)070 [SPIRES] [hep-ph/0408248]

[17] Arkani-Hamed N and Dimopoulos S, 2005 J. High Energy Phys. JHEP06(2005)073 [SPIRES] [hep-th/0405159]

[18] Reno M H, Sarcevic I and Su S, 2005 Astropart. Phys. 24107 [SPIRES] [hep-ph/0503030]

[19] Huang Y, Reno M H, Sarcevic I and Uscinski J, 2006 Phys. Rev. D 74115009 [SPIRES] [hep-ph/0607216]

[20] Giudice G F and Rattazzi R, 1999 Phys. Rep. 322419 [SPIRES] [hep-ph/9801271]

[21] Albuquerque I, Burdman G and Chacko Z, 2004 Phys. Rev. Lett. 92 221802 [SPIRES] [hep-ph/0312197]

[22] Bi X-J, Wang J-X, Zhang C and Zhang X, 2004 Phys. Rev. D 70123512 [SPIRES] [hep-ph/0404263]

[23] Ahlers M, Kersten J and Ringwald A, 2006 J. Cosmol. Astropart. Phys. JCAP07(2006)005 [SPIRES] [hep-ph/0604188]

[24] Albuquerque I F M, Burdman G and Chacko Z, 2007 Phys. Rev. D 75035006 [SPIRES] [hep-ph/0605120]

[25] Illana J I, Masip M and Meloni D, 2006 Proc. Exotic Physics with Neutrino Telescopes (Uppsala, Sept. 2006) [hep-ph/0612305]

[26] Heck D, Schatz G, Thouw T, Knapp J and Capdevielle J N, CORSIKA: A Monte Carlo code to simulate extensive air showers, 1998 Report FZKA-6019 Forschungszentrum Karlsruhe http://www-ik.fzk.de/corsika

[27] Dawson S, Eichten E and Quigg C, 1985 Phys. Rev. D 311581 [SPIRES]

[28] Yao W M et al (Particle Data Group), 2006 J. Phys. G: Nucl. Part. Phys. 331 [SPIRES]

[29] Abazov V M et al (D0), 2006 Phys. Lett. B 638119 [SPIRES] [hep-ex/0604029]

[30] Pumplin J et al, 2002 J. High Energy Phys. JHEP07(2002)012 [SPIRES] [hep-ph/0201195]

[31] Sutton P J, Martin A D, Roberts R G and Stirling W J, 1992 Phys. Rev. D 452349 [SPIRES]

[32] Beenakker W, Hopker R, Spira M and Zerwas P M, 1997 Nucl. Phys. B 49251 [SPIRES] [hep-ph/9610490]

[33] Eidelman S et al (Particle Data Group), 2004 Phys. Lett. B 5921 [SPIRES]

[34] Gandhi R, Quigg C, Reno M H and Sarcevic I, 1996 Astropart. Phys. 581 [SPIRES] [hep-ph/9512364]

[35] Ribordy M, 2007 Nucl. Instrum. Methods A 574137 [astro-ph/0611604] 